\title{
High power microwave devices: development since 1880
}

\begin{abstract}
High power microwave systems have emerged as a promising new technology that has many applications, which include high power radar, directed energy weapons, laboratory sources for susceptibility and vulnerability testing of electronic systems. These systems are built, applied, and studied in many developed countries such as in United States of America and China. In the recent years, other countries such as Russia, Western Europe, Japan, Taiwan, India, South Korea, and Singapore have also entered the research spheres. In this paper an introduction to the emergence of HPM and the sequential evolution of the technology, that plays an important role in several applications, are discussed. The discussion extends to types of HPM sources, and their effects of electromagnetic interference on electronic systems.
\end{abstract}

Keyword: High power microwaves; HPM; Electromagnetic pulse; EMP; Electromagnetic interference; EMI 